\title{
Correspondence between the RAND Negative Impact of Asthma on Quality of Life Item Bank and the Marks Asthma Quality of Life Questionnaire
}

\author{
Maria Orlando Edelen, $\mathrm{PhD}^{1}$, Brian D. Stucky, $\mathrm{PhD}^{2}$, Cathy Sherbourne, $\mathrm{PhD}^{2}$, Nicole \\ Eberhart, $\mathbf{P h D}^{2}$, and Marielena Lara, MD, MPH ${ }^{2}$ \\ ${ }^{1}$ RAND Corporation, Boston, MA, 02116, USA \\ ${ }^{2}$ RAND Corporation, Santa Monica, CA, 90407, USA
}

\begin{abstract}
Purpose-In many research and clinical settings where patient reported outcomes (PRO) measures are used, it is often desirable to link scores across disparate measures, or use scores from one measure to describe scores on a separate measure. However, PRO measures are scored using a variety of metrics, making such comparisons difficult. In this paper, we provide an example of how to transform scores across disparate measures (the Marks Asthma Quality of Life Questionnaire (AQLQ-Marks) and the newly developed RAND-Negative Impact of Asthma on Quality of Life item bank (RAND-IAQL-Bank)) using an IRT-based linking method
\end{abstract}

Methods-Our sample of adults with asthma ( $\mathrm{N}=2032)$ completed two measures of asthmaspecific quality of life: the AQLQ-Marks and the RAND-IAQL-Bank. We use item response theory- (IRT) based co-calibration of the two measures to provide a linkage, or a common metric between the two. Co-calibration refers to the process of using IRT to estimate item parameters that describe the responses to the scales' items according to a common metric, in this case a normal distribution transformed to a T-scale with a mean of 50 and a standard deviation of 10 .

Findings-Results indicated that the scales measured highly similar constructs and were sufficiently unidimensional for IRT co-calibration. Once linked, scores from the two measures were invariant across subgroups. A crosswalk is provided that allows researchers and clinicians using AQLQ-Marks to crosswalk to the RAND-IAQL toolkit.

Implications-The ability to translate scores from the RAND-IAQL toolkit to other 'legacy' measures increases the value of the new toolkit, aids in interpretation, and hopefully will facilitate adoption by asthma researchers and clinicians. More generally, the techniques we illustrate can be

(C) 2014 Excerpta Medica, Inc. All rights reserved.

Please direct correspondence to Maria Orlando Edelen, PhD., 20 Park Plaza, Suite 920, Boston, MA 02116; orlando@ rand.org; phn: (617) 338-2059 x8634; fax: (617) 357-7470

Publisher's Disclaimer: This is a PDF file of an unedited manuscript that has been accepted for publication. As a service to our customers we are providing this early version of the manuscript. The manuscript will undergo copyediting, typesetting, and review of the resulting proof before it is published in its final citable form. Please note that during the production process errors may be discovered which could affect the content, and all legal disclaimers that apply to the journal pertain.

CONFLICT OF INTEREST

There are no conflicts of interest to disclose. The content is solely the responsibility of the authors and does not necessarily represent the official views of the National Institutes of Health. 
applied to other newly developed or existing measures in the PRO research field to obtain crosswalks with widely used traditional, legacy instruments.

\section{Keywords}

Asthma; Quality of Life; IRT; item bank; RAND-IAQL; AQLQ-Marks; linking

\section{Introduction}

In response to recommendations from the Asthma Quality of Life Subcommittee of the 2010 NIH Asthma Outcomes Workshop, we developed a freely available new toolkit of measures for assessing the impact of asthma on a patient's quality of life (QoL) that focuses on the patient's perception of the impact or bother of asthma on his or her QoL. Developed using item response theory (IRT), the new system consists of an item bank (the RAND-Negative Impact of Asthma on Quality of Life item bank (RAND-IAQL-Bank)), and 4 and 12 item short forms (RAND-IAQL-4, RAND-IAQL-12). These scales avoid confounding QoL with asthma symptomatology and functional impairment while including content ranging across many domains of life important to people with asthma based on their own voice. ${ }^{1,2}$

Over the years, other standard asthma-specific quality of life instruments have been used in clinical trials, for population monitoring and for guidance in decision-making regarding asthma treatment. ${ }^{3-12}$ However, because most of these commonly used or "legacy" measures tend to confound QoL with asthma symptoms, functional impairment, and control, none were recommended as "core" or required for federally sponsored asthma clinical research by the Asthma Outcomes Workshop or in their subsequent review article. ${ }^{13}$

In response, our new measurement system focuses specifically on patient-reported asthma QoL. Items do not appear to confound QoL with asthma symptomatology, control, or functional impairment and there is preliminary evidence of the construct validity of our measures. ${ }^{2,14}$ However, because other asthma-specific QoL measures have been used widely over the years, researchers' and clinicians may be hesitant to adopt a new measure that has little comparison data available to aid interpretation of results. For example, healthcare providers or those conducting clinical trials who have identified meaningful cut scores on existing measures (e.g., to highlight clinical relevance, indicate special attention for asthma treatment, identify promising new therapeutics, etc.) will want information on how our new measures correspond to the legacy scores.

To address this need, this paper describes the correspondence between scores on one legacy measure, the Marks Asthma Quality of Life Questionnaire (AQLQ-Marks ${ }^{3,11}$ ) and the RAND-IAQL-Bank ${ }^{2}$. The process we use, referred to as score linking is the process of transforming scores across disparate measures. ${ }^{15}$ This paper describes one particular technique, IRT-based calibration, which uses item response theory (IRT) methodology to provide a common metric for scores from two measures (i.e., the RAND-IAQL-Bank and AQLQ-Marks). Calibration refers to the process of using IRT to estimate item parameters that describe the responses to the scales' items according to a common metric, in this case a normal distribution transformed to have a mean of 50 and a standard deviation of 10 . A result of this is that once calibrated, scaled scores may be computed from either the AQLQ- 
Marks or any set of items from the RAND-IAQL-Bank, and the resulting scores will equally reflect the negative impact of asthma on QoL regardless of which measure respondents completed.

In what follows, we first describe our sample and data collection, and then provide more detail for the analytic approach. Results are presented that substantiate the suitability of the linking approach, provide information about the characteristics of AQLQ-Marks items relative to items in the RAND-IAQL-Bank, and delineate a scoring crosswalk between the AQLQ-Marks and the RAND-IAQL-Bank.

\section{Methods}

\section{Sample description}

A national sample of adults (ages 18+) with asthma ( $\mathrm{N}=2032)$ completed an internet-based survey assessment hosted by Harris Interactive. Eligible participants satisfied two conditions: (1) a doctor or other health professional had informed them that they had asthma, and (2) they reported still having asthma. In addition to ensure response variation across the range of asthma severity we required that $90 \%$ of the sample experienced at least one episode of asthma or an asthma attack during the prior 12 months. ${ }^{16}$ Table 1 describes the demographic characteristics and health service utilization patterns of participants, and additional sampling details are available in Stucky et al. ${ }^{2}$

\section{Measures}

RAND-Negative Impact of Asthma on Quality of Life (RAND-IAQL toolkit)—The RAND-IAQL toolkit was developed using both qualitative and quantitative methods. Prior to psychometric testing, an initial 112-item pool was established using focus groups, cognitive testing, expert review, and literature review (see Eberhart et al. ${ }^{1}$ for a detailed description of the item development process). After field testing the item pool, quantitative analyses were conducted using factor analysis and IRT. These analyses were used to reduce the item set to one that was strongly unidimensional. For example, based on factor analysis results, we identified and examined locally dependent item clusters and removed items based on empirical and subjective (e.g., content) information to eradicate the dependence. This process ultimately resulted in a final 65 -item bank. Assessments of dimensionality presented in Stucky et al. ${ }^{2}$ suggest that the set of items measure a single construct referred to as the negative impact of asthma on QoL. The 65-item unidimensional bank was calibrated with IRT using the graded response model $(\mathrm{GRM})^{17}$ (marginal reliability $=0.98$ ). Among the 65-items, brief 4- and 12-item short forms were developed that focus on the global aspects of asthma-specific QoL (RAND-IAQL-4; marginal reliability $=0.86$ ) and broadened content coverage (RAND-IAQL-12; marginal reliability $=0.93$ ). Items in the bank have a consistent timeframe (past 4-weeks), orientation (first-person), and response format (5-point categorical) reflecting magnitude (i.e., "not at all" to "very much") or frequency of impact (i.e., "never" to "almost always"). Scores for all RAND-IAQL measures have been transformed to a T-score metric with a mean of 50 and a standard deviation of 10; higher scores on the RAND-IAQL item sets indicate greater negative impact of asthma on QoL. 
Marks Asthma Quality of Life Questionnaire-The Marks Asthma Quality of Life Questionnaire (AQLQ-Marks) includes 20 questions yielding a total QoL score and 4 subscale scores: breathlessness (which includes breathlessness and physical restrictions), concern (related to concerns about health), mood disturbance, and social disruption. ${ }^{11,18}$ Each item has five response options ranging from "not at all" to "very severely" and is scored on a 0-4 scale. The total score is calculated as the mean of the 20 item scores multiplied by 2.5 (range $0-10$ ). Higher scores indicate a more negative impact of asthma on quality of life. Studies have provided evidence for the cross-sectional construct validity and reproducibility of this measure, ${ }^{11,19}$ it has also been shown to predict asthma severity, work days missed and to be responsive to changes in pulmonary function. ${ }^{3}$ It has been used in a number of studies over the years within and outside the United States. ${ }^{3,5,20-29}$

\section{Approach}

A necessary condition for IRT linking is that the scales to be linked measure the same or highly similar constructs; therefore, to determine whether the RAND-IAQL-Bank and AQLQ-Marks were sufficiently associated so as to be appropriate for IRT co-calibration, we first confirmed that the correlation between the two total scores was large enough (a common rule of thumb is that the correlation should be about 2.8$).{ }^{15} \mathrm{Next}$, to establish essential unidimensionality of the combined set of 85 items (65 RAND-IAQL-Bank items and 20 AQLQ-Marks items), we conducted exploratory factor analysis (EFA) of the combined item sets using Mplus software ${ }^{30}$ and the mean and variance adjusted weighted least squares algorithm (WLSMV) that is appropriate for categorical response items. We evaluated dimensionality based on the number of eigenvalues greater than 1 , the ratio of the first to second eigenvalue, examination of the scree plot, strength of the factor loadings, overall fit of the 1 -factor model using established indices (i.e., RMSEA $\leq 08$, TLI $\geq 95$, CFI $\geq$ 95), ${ }^{31}$ and extent of interpretability of the two factor solution.

Next we conducted an IRT calibration of the entire 85 -item set using IRTPRO ${ }^{32}$ and the graded response model (GRM) ${ }^{17}$ For this calibration, because we are interested in generating a crosswalk to the RAND-IAQL-Bank scores as they are intended to be used in research, the item parameters for the RAND-IAQL-Bank were fixed to their published values $^{2}$ and parameters were estimated for each AQLQ-Marks item relative to the established RAND-IAQL-Bank parameters. The suitability of the model was evaluated primarily through examination of the AQLQ-Marks parameter estimates and standard errors, making sure estimates were within the expected range and adequately precise. Based on results of this co-calibration, we generated graphs of the magnitude of the item discrimination and average location parameter estimates to illustrate the extent of correspondence between the two instruments.

One important threat to the validity of a test linkage is the possibility that the comparability does not apply across different sub-group populations (i.e., lack of invariance at sub-group level). Fortunately, there is a straightforward method to detect such lack of invariance empirically. Following the approach employed by Thissen et al (2011), ${ }^{33}$ we calculated the root mean squared deviation (RMSD) statistic to check invariance of subgroup differences in linked scores according to gender, minority status, education, COPD comorbidity, 
instance of an overnight hospital stay in the past year, and occurrence of an asthma attack in the past year. Dorans and Holland (2000) have suggested that RMSD values of 1-8\% have been found to be associated with useful linkages. ${ }^{15}$ Finally, we used the AQLQ-Marks item parameters from the IRT co-calibration results to generate an AQLQ-Marks summed score to IRT T-score translation table.

\section{Results}

The correlation between the RAND-IAQL-Bank score and the AQLQ-Marks total score was .90, and EFA results indicated that the RAND-IAQL-Bank and AQLQ-Marks items were sufficiently unidimensional for IRT co-calibration. Although the EFA yielded four eigenvalues greater than one, the ratio of the first to second eigenvalue and the scree plot clearly indicated 1 factor $(60.67,2.3,1.4,1.3,0.9,0.8,0.7,0.6, \ldots)$. Furthermore, the fit of the 1 factor model met established criteria $(\mathrm{CFI}=0.955, \mathrm{TLI}=0.954$, and $\mathrm{RMSEA}=0.063)$, all item loadings were strong, positive and significant (range: .73 - .93), and the two factor solution did not extract a substantively meaningful second factor. With this strong evidence for essential unidimensionality, we were able to proceed with IRT co-calibration.

The IRT co-calibration using established RAND-IAQL-Bank parameters yielded AQLQMarks item parameter estimates that are comparable in magnitude to RAND-IAQL-Bank item parameters. Figure 1 displays the rank order of the item discrimination parameters for the entire 85-item set (higher values indicate a stronger association with the underlying construct represented by the item set). The full range of discrimination parameters is $1.8-$ 4.2, and the AQLQ-Marks items (shown as squares with variable fill depending on subscale) are distributed throughout that range. It is noteworthy that items from the AQLQ-Marks Social subscale tend to have discrimination parameters on the higher end of the range indicating higher similarity with the RAND-IAQL-Bank items whereas items from the Breathlessness subscale tend to be on the lower end of the range and thus less similar to the RAND-IAQL-Bank item content. Figure 2 provides comparable information using the average item location parameters. The IRT calibration yielded a total of four location parameters for each item indicating the spacing of the item response categories along the impact of asthma on QoL continuum. The location parameters indicate where on the impact continuum a person is likely to be if they endorse a given response option, and the average location parameter value can be interpreted as an indicator of the overall severity of the item. As with the discrimination parameters, the average location parameters for the AQLQMarks items are fairly evenly distributed throughout the range that extends from .32-1.0. There are no discernible patterns for these values according to AQLQ-Marks subscale.

To check the suitability of the linkage, we calculated group mean differences in AQLQMarks and IAQL-Bank scores according to gender, minority status (non-Hispanic white vs. non-white), education (high school diploma or less vs. some college or more), COPD comorbidity, and indicators of an overnight hospital stay and an asthma attack in the past 12 months. The group mean differences are displayed in Table 2 as effect sizes and the discrepancy in these mean difference estimates is reflected in the RMSD. As can be seen from the table, the standardized differences are very similar across grouping variables and 
all RMSD values are well below the suggested threshold of $8 \%$, lending support for the robustness of the linkage between the two scales.

Table 3 provides a score conversion table to convert AQLQ-Marks total scores to the RAND-IAQL toolkit T-score metric.

\section{Discussion}

The RAND-IAQL toolkit offers a psychometrically sound method for assessing asthmaspecific QoL. The toolkit was developed in response to task force suggestions to emphasize the patient perspective and distinguish burden of asthma from symptoms, functioning and control. This article presents a crosswalk from RAND-IAQL-Bank scores to scores on one 'legacy' measure, the AQLQ-Marks. Results strongly suggest that the necessary conditions for IRT linking were met. The scales measured highly similar constructs and were sufficiently unidimensional for IRT co-calibration. Furthermore, IRT item parameters for the AQLQ-Marks items were within the expected range and estimates were adequately precise. Follow-up analyses convincingly demonstrated that linked scores were invariant across demographic and health service use subgroups.

It is interesting to note that while the RAND-IAQL-Bank and AQLQ-Marks are sufficiently unidimensional and measure highly similar constructs, the RAND-IAQL-Bank has more items measuring severe aspects of the impact of asthma on QoL as evidenced by the comparison of item parameters across measures. The average location parameters for RAND-IAQL-Bank items are more widely distributed across the negative impact continuum than those for the AQLQ-Marks. In addition, the AQLQ-Marks Breathlessness subscale items tend to be on the lower end of the discrimination parameter range suggesting that the Marks AQLQ items with symptom content are less related to the RAND-IAQL-Bank items than are non-symptom content items.

The ability to crosswalk scores from the RAND-IAQL to 'legacy' measures increases the value of the new measure, aids in interpretation, and hopefully will facilitate adoption by asthma researchers and clinicians. As an example of the utility of the crosswalk, consider Dean et al., $2009^{22}$ who classified participants in their study as having uncontrolled or controlled asthma based on a treatment and symptom guideline-based algorithm. Adult uncontrolled asthmatics in that study had AQLQ-Marks median scores of 3.6 and controlled asthmatics had AQLQ-Marks median scores of 2.1. These translate to scores of about 52.8 (uncontrolled) and 47.7 (controlled) on the RAND-IAQL-Bank T-score scale. A separate study of adults with asthma reports an overall sample mean of $2.0,{ }^{34}$ which translates to a score of 47.2 on the RAND-IAQL-Bank T-score scale. This study also provided mean AQLQ-Marks scores for four subgroups defined based on perceived asthma severity. Using the scoring translation table developed here, the subgroup AQLQ-Marks scores 'crosswalked' to the RAND-IAQL-Bank T-score scale are as follows: No symptoms ( 0.575 to 39.7), Mild (1.325 to 44.4), Moderate (1.825 to 46.7), and Severe (3.95 to 54.0). Using the crosswalk illustrates the relatively large difference in severity between the "Moderate" and "Severe" groups, and indicates that this sample has somewhat less severe asthma than our calibration sample (the translated overall score of 47.2 is about .3 SDs below the 
RAND-IAQL-Bank mean of 50). It should be noted that the crosswalk presented in Table 3 is based on the original recommended $0-10$ scoring method recommended by Marks. If, as in the examples presented here, the AQLQ-Marks scores are reported on a 0-4 scale it is necessary to first transform the AQLQ-Marks score to the $0-10$ scale (by multiplying by 2.5) to get comparable scores for the crosswalk.

Future studies can use similar methods to obtain crosswalks for other legacy instruments in the PRO research field. The co-calibration approach we describe, and similar IRT-based approaches, can be used to apply IRT-based scoring methods to newly-developed PRO measures while maintaining comparability with traditional, legacy measures. IRT-based measurement offers many advantages over traditional methods. The ability to provide a bridge from new measures to commonly used 'legacy' instruments facilitates comparisons across time and across studies and can enhance the utility of both existing and new instruments as patient-centered research moves forward.

\section{Acknowledgments}

Research reported in this publication was supported by the National Heart, Lung, and Blood Institute of the National Institutes of Health under Award Number R01HL107312.

\section{References}

1. Eberhart NK, Sherbourne CD, Edelen MO, Stucky BD, Sin NL, Lara M. Development of a measure of asthma-specific quality of life among adults. Quality of Life Research. 2013:1-12.

2. Stucky BD, Edelen MO, Sherbourne CD, Eberhart NK, Lara M. Developing an item bank and short forms that assess the impact of asthma on quality of life. Respiratory medicine. 2013

3. Adams RJ, Ruffin RE, Smith BJ. Validity of a modified version of the Marks Asthma Quality of Life Questionnaire. Journal of Asthma. 2000; 37(2):131-143. [PubMed: 10805202]

4. Barley E, Quirk F, Jones P. Asthma health status measurement in clinical practice: validity of a new short and simple instrument. Respiratory medicine. 1998; 92(10):1207-1214. [PubMed: 9926151]

5. Bayliss MS, Espindle DM, Buchner D, Blaiss MS, Ware JE. A new tool for monitoring asthma outcomes: the ITG Asthma Short Form. Quality of Life Research. 2000; 9(4):451-466. [PubMed: 11131937]

6. Hyland M. The living with asthma questionnaire. Respiratory medicine. 1991; 85:13-16. [PubMed: 1759015]

7. Hyland ME, Ley A, Fisher DW, Woodward V. Measurement of psychological distress in asthma and asthma management programmes. British journal of clinical psychology. 1995; 34(4):601-611. [PubMed: 8563667]

8. Jones PW, Quirk FH, Baveystock CM, Littlejohns P. A self-complete measure of health status for chronic airflow limitation: the St. George's Respiratory Questionnaire. American Review of Respiratory Disease. 1992; 145(6):1321-1327. [PubMed: 1595997]

9. Juniper E, Guyatt G, Cox F, Ferrie P, King D. Development and validation of the mini asthma quality of life questionnaire. European Respiratory Journal. 1999; 14(1):32-38. [PubMed: 10489826]

10. Juniper EF, Guyatt G, Epstein RS, Ferrie PJ, Jaeschke R, Hiller TK. Evaluation of impairment of health related quality of life in asthma: development of a questionnaire for use in clinical trials. Thorax. 1992; 47(2):76-83. [PubMed: 1549827]

11. Marks GB, Dunn SM, Woolcock AJ. An evaluation of an asthma quality of life questionnaire as a measure of change in adults with asthma. Journal of clinical epidemiology. 1993; 46(10):11031111. [PubMed: 8410095] 
12. Schatz M, Mosen D, Kosinski M, et al. Validation of the asthma impact survey, a brief asthmaspecific quality of life tool. Quality of Life Research. 2007; 16(2):345-355. [PubMed: 17033905]

13. Wilson SR, Rand CS, Cabana MD, et al. Asthma outcomes: Quality of life. Journal of Allergy and Clinical Immunology. 2012; 129(3):S88-S123. [PubMed: 22386511]

14. Sherbourne CD, Stucky BD, Edelen MO, Eberhart NK, Kleeup E, Lara M. Assessing the validity of the RAND Negative Impact of Asthma on Quality of Life Short-Forms. Journal of Allergy and Clinical Immunology. in press.

15. Dorans NJ, Holland PW. Population invariance and the equatability of tests: Basic theory and the linear case. Journal of Educational Measurement. 2000; 37(4):281-306.

16. Center for Disease Control. National Center for Environmental Health (NCEH): Division of Environmental Hazards and Health Effects. America Breathing Easier 2010: CDC's National Asthma Control Program at a Glance. 2010

17. Samejima, F. Graded response model. In: Hambleton, WJvdLaRK., editor. Handbook of modern item response theory. New York: Springer-Verlag; 1997. p. 85-100.

18. Marks GB, Dunn SM, Woolcock AJ. A scale for the measurement of quality of life in adults with asthma. Journal of clinical epidemiology. 1992; 45(5):461-472. [PubMed: 1588352]

19. Katz PP, Eisner MD, Henke J, Shiboski S, Yelin EH, Blanc PD. The Marks Asthma Quality of Life Questionnaire: further validation and examination of responsiveness to change. Journal of clinical epidemiology. 1999; 52(7):667-675. [PubMed: 10391660]

20. Armour C, Bosnic-Anticevich S, Brillant M, et al. Pharmacy Asthma Care Program (PACP) improves outcomes for patients in the community. Thorax. 2007; 62(6):496-592. [PubMed: 17251316]

21. Blanc PD, Cisternas M, Smith S, Yelin EH. Asthma, employment status, and disability among adults treated by pulmonary and allergy specialists. CHEST Journal. 1996; 109(3):688-696.

22. Dean BB, Calimlim BM, Kindermann SL, Khandker RK, Tinkelman D. The impact of uncontrolled asthma on absenteeism and health-related quality of life. Journal of Asthma. 2009; 46(9):861-866. [PubMed: 19905909]

23. Eisner MD, Boland M, Tolstykh I, Mendoza G, Iribarren C. Intensive care unit admission for asthma: a marker for severe disease. Journal of Asthma. 2005; 42(5):315-323. [PubMed: 16036406]

24. Katz PP, Yelin EH, Eisner MD, Earnest G, Blanc PD. Performance of valued life activities reflected asthma-specific quality of life more than general physical function. Journal of clinical epidemiology. 2004; 57(3):259-267. [PubMed: 15066686]

25. King MT, Kenny PM, Marks GB. Measures of asthma control and quality of life: longitudinal data provide practical insights into their relative usefulness in different research contexts. Quality of Life Research. 2009; 18(3):301-312. [PubMed: 19225906]

26. Marks G, Burney P, Premaratne U, Simpson J, Webb J. Asthma in Greenwich, UK: impact of the disease and current management practices. European Respiratory Journal. 1997; 10(6):1224-1229. [PubMed: 9192920]

27. Osborne ML, Vollmer WM, Linton KL, Buist SA. Characteristics of patients with asthma within a large HMO: a comparison by age and gender. American journal of respiratory and critical care medicine. 1998; 157(1):123-128. [PubMed: 9445289]

28. Storms B, Olden L, Nathan R, Bodman S. Effect of allergy specialist care on the quality of life in patients with asthma. Annals of allergy, asthma \& immunology: official publication of the American College of Allergy, Asthma, \& Immunology. 1995; 75(6 Pt 1):491.

29. Vollmer WM, O'Hollaren M, Ettinger KM, et al. Specialty differences in the management of asthma: a cross-sectional assessment of allergists' patients and generalists' patients in a large HMO. Archives of internal medicine. 1997; 157(11):1201. [PubMed: 9183231]

30. Muthén L, Muthén B. Mplus user's guide, 1990-2010. 2011

31. Hu, Lt; Bentler, PM. Cutoff criteria for fit indexes in covariance structure analysis: Conventional criteria versus new alternatives. Structural Equation Modeling: A Multidisciplinary Journal. 1999; 6(1):1-55.

32. Cai, L.; Du Toit, S.; Thissen, D. IRTPRO: Flexible, multidimensional, multiple categorical IRT modeling [Computer software]. Chicago, IL: Scientific Software International; 2011. 
33. Thissen D, Varni JW, Stucky BD, Liu Y, Irwin DE, DeWalt DA. Using the PedsQL ${ }^{\text {TM }} 3.0$ asthma module to obtain scores comparable with those of the PROMIS pediatric asthma impact scale (PAIS). Quality of Life Research. 2011; 20(9):1497-1505. [PubMed: 21384264]

34. Apfelbacher CJ, Jones C, Hankins M, Smith H. Validity of two common asthma-specific quality of life questionnaires: Juniper mini asthma quality of life questionnaire and Sydney asthma quality of life questionnaire. Health and quality of life outcomes. 2012; 10(1):1-9. [PubMed: 22208808] 


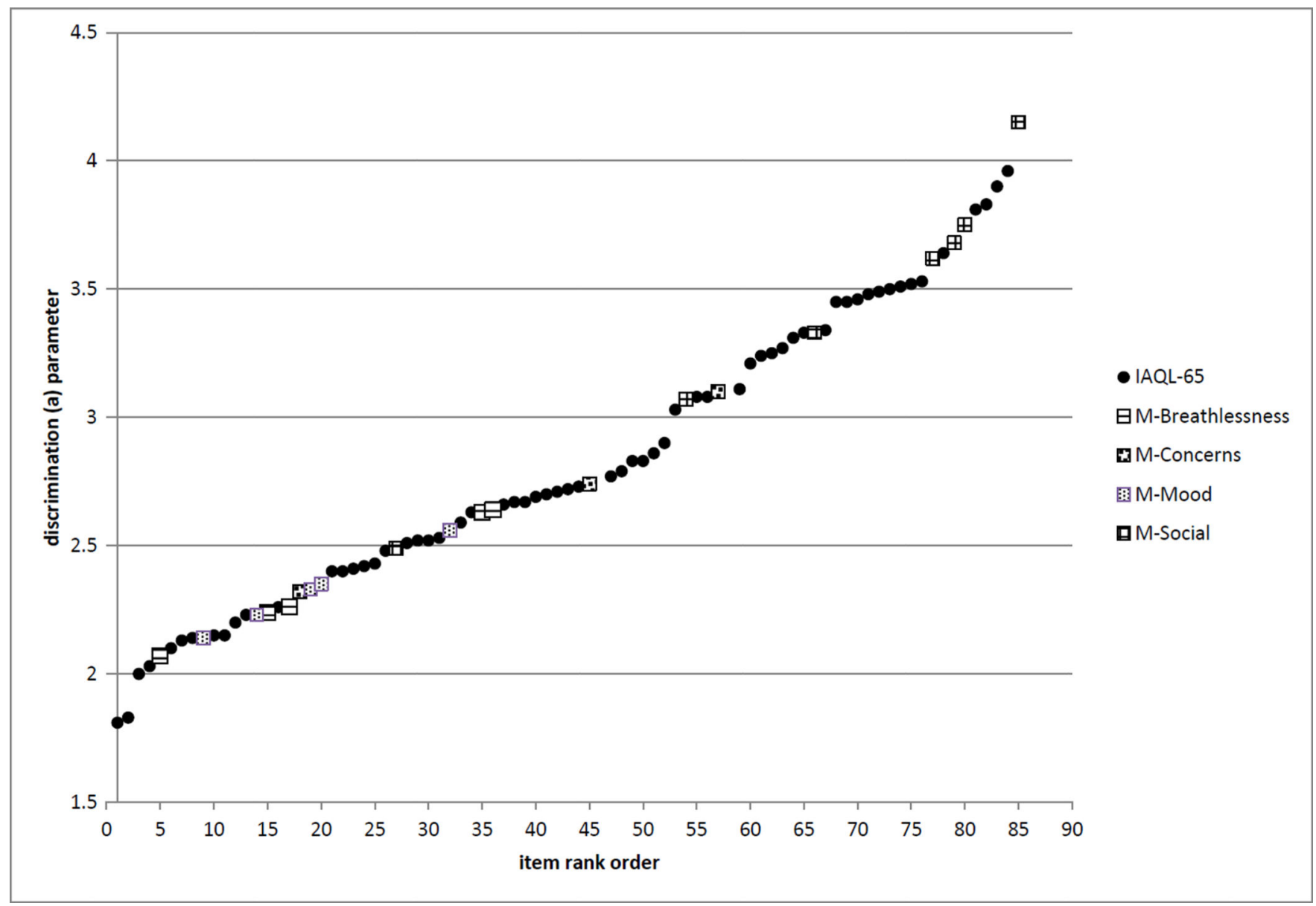

Figure 1. Discrimination (a) parameters for RAND-IAQL-BANK and AQLQ-Marks items Items from the two sources are ordered on the $\mathrm{X}$-axis according to the magnitude of the discrimination parameter (y-axis). RAND-IAQL-Bank items are indicated as dark circles, AQLQ-Marks items are indicated as squares with variable fill depending on AQLQ-Marks subscale. 


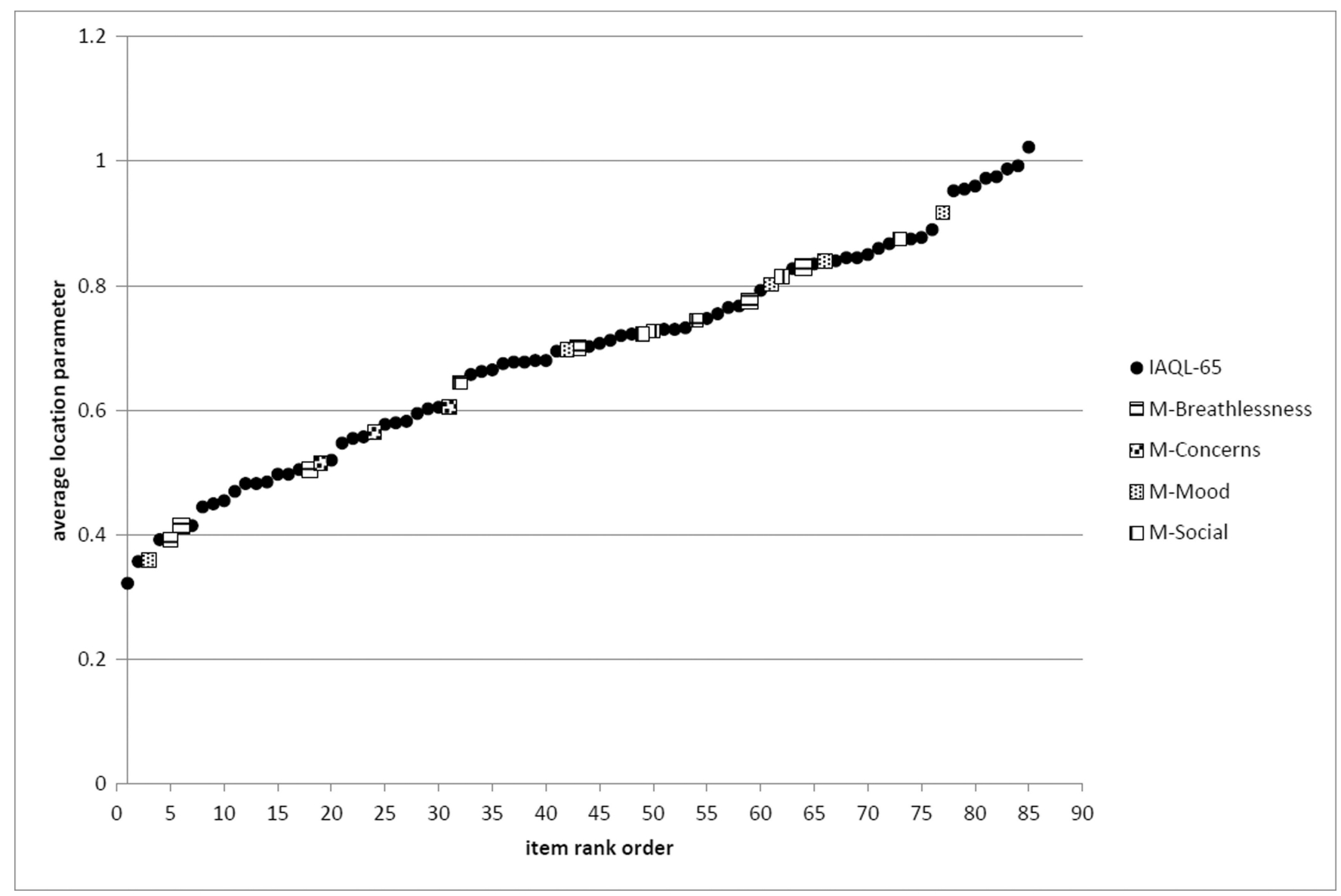

Figure 2. Average Location (b) parameters for RAND-IAQL-BANK and AQLQ-Marks items Items from the two sources are ordered on the $\mathrm{X}$-axis according to the magnitude of the average location parameter (y-axis). RAND-IAQL-Bank items are indicated as dark circles, AQLQ-Marks items are indicated as squares with variable fill depending on AQLQ-Marks subscale. 
TABLE 1

Demographic Characteristics of Asthma Sample (N=2032)

\begin{tabular}{|c|c|}
\hline & Mean or $\mathrm{N}(\%$ or SD $)$ \\
\hline Mean Age (SD) & $43(15)$ \\
\hline \multicolumn{2}{|l|}{ Gender } \\
\hline female & $1219(60)$ \\
\hline \multicolumn{2}{|l|}{ Ethnicity/Race } \\
\hline Hispanic & 282(14) \\
\hline Asian & $221(11)$ \\
\hline African American & $381(19)$ \\
\hline Non-Hispanic White & $1148(56)$ \\
\hline \multicolumn{2}{|l|}{ Region of US } \\
\hline East & $478(24)$ \\
\hline Midwest & $432(21)$ \\
\hline South & $578(28)$ \\
\hline West & 544(27) \\
\hline \multicolumn{2}{|l|}{ Education } \\
\hline$<=$ High School & $353(17)$ \\
\hline Some college & $743(36)$ \\
\hline College Graduate & $490(24)$ \\
\hline some Graduate school or grad degree & $446(22)$ \\
\hline \multicolumn{2}{|l|}{ Medical Comorbidities } \\
\hline Sinusitis & $1284(63)$ \\
\hline Diabetes & $505(25)$ \\
\hline Heart Disease & 194(9) \\
\hline COPD & $287(14)$ \\
\hline Migraine headaches & $660(32)$ \\
\hline Hospital Stay past year & $385(19)$ \\
\hline Mean number of Emergency visits for Asthma in past year (SD) & $1.35(6.60)$ \\
\hline \multicolumn{2}{|l|}{ Self-Rating of Asthma Severity } \\
\hline Very mild & $383(19)$ \\
\hline Mild & $580(28)$ \\
\hline Moderate & $824(41)$ \\
\hline Severe & 207(10) \\
\hline Very Severe & $36(2)$ \\
\hline \multicolumn{2}{|l|}{ Frequency used rescue inhaler past 4 weeks } \\
\hline Never & $495(24)$ \\
\hline 1 time per week & $510(25)$ \\
\hline 2 or more times per week but not daily & $600(29)$ \\
\hline Daily & 279(14) \\
\hline
\end{tabular}




\begin{tabular}{|l|c|}
\hline & Mean or N(\% or SD) \\
\hline Several times a day most days & $143(7)$ \\
\hline Used controller medication daily in the past 4 weeks & $1163(57)$ \\
\hline Asthma Control Test level & \\
\hline Controlled (<10) & $770(38)$ \\
\hline Somewhat Controlled (15-19) & $446(22)$ \\
\hline Poorly Controlled (20+) & $816(40)$ \\
\hline
\end{tabular}


TABLE 2

Standardized Group Mean Difference Estimates and RMSD for AQLQ-Marks and RAND-IAQL-BANK Scores

\begin{tabular}{lccc}
\hline \multicolumn{4}{c}{ Standardized difference } \\
\hline Grouping Variable & AQLQ-Marks & RAND-IAQL-BANK & RMSD \\
\hline Gender & 0.15 & 0.17 & $1.06 \%$ \\
Minority Status & 0.51 & 0.54 & $1.78 \%$ \\
Education & 0.28 & 0.28 & $0.13 \%$ \\
COPD & 0.73 & 0.69 & $1.61 \%$ \\
Hospital Stay & 1.35 & 1.36 & $0.41 \%$ \\
Asthma Attack & 1.05 & 1.12 & $3.25 \%$ \\
\hline
\end{tabular}

Note: RMSD is root mean squared difference; minority status grouping is non-Hispanic white vs. non-white; education is high school or less vs. some college or higher; COPD, Hospital stay and Asthma attack groupings are yes vs. no. 


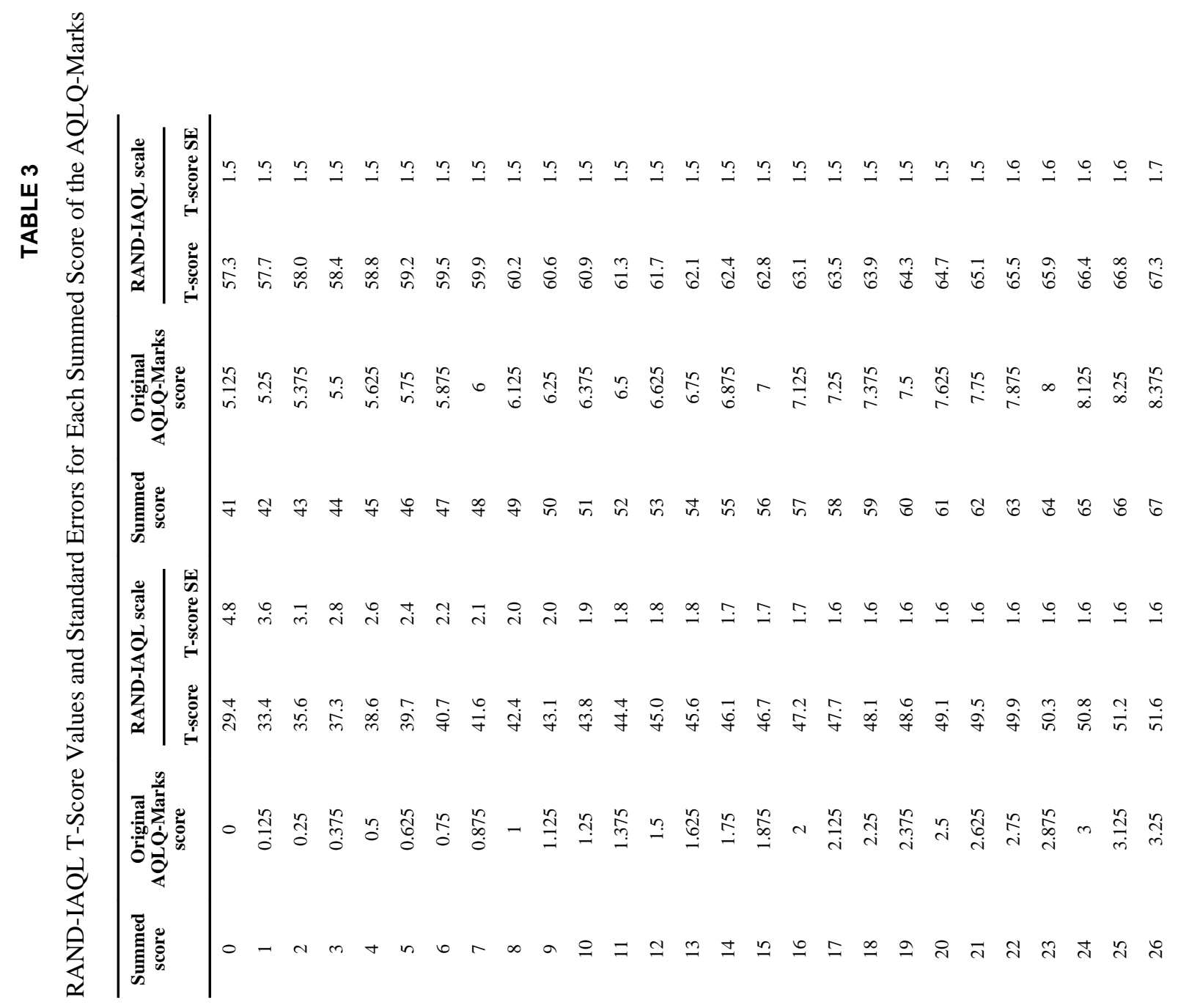




|

